\title{
VARIABILIDAD DE LA PRECIPITACIÓN ANUAL ASOCIADA CON LOS EVENTOS EL NIÑO YLANIÑA EN LAS CIUDADES DE NARANJO, PALMARES Y SAN RAMÓN, COSTA RICA
}

\author{
Marvin E. QUESADA ${ }^{1}$ \\ Universidad de Costa Rica
}

\begin{abstract}
RESUMEN: Se investiga el efecto del evento ENOS (El Niño/La Niña-Oscilación del Sur) en la precipitación anual de las ciudades de Naranjo, Palmares y San Ramón ubicados en el sector extremo oeste de la Depresión Tectónica Central de Costa Rica. Para la ciudad de Naranjo se cuenta con un total de 30, Palmares 28 y San Ramón 42 años. Los resultados muestran que durante la permanencia del evento El Niño los promedios de precipitación anual son bajos mientras que durante los eventos La Niña los promedios son superiores. A pesar de ello no existe un patrón temporal ni similitud espacial entre dichas ciudades, tal es el caso de Naranjo que muestra años muy húmedos durante la fase fría, pero no registra una correspondencia marcada durante los años de la fase cálida. En Palmares la precipitación tiende más a las mermas o años secos durante El Niño, mientras que San Ramón pareciera tener una alta correspondencia con ambas fases.

PALABRAS CLAVE: Precipitación anual, ENOS, Fase Fría, Fase Caliente, variación geográfica.

ABSTRACT: This study investigates the effect of ENSO (El Niño and La Niña Southern Oscillation) on the annual precipitation. The city of Naranjo has 30, Palmares 28 and San Ramón 42 years or record. All the cities are located in the extreme western sector of the Central Tectonic Depression of Costa Rica. The results demonstrate that during years of El Niño, the annual average precipitation is low but during years of La Niña, the average precipitation increases in all three cities, in spite of the fact that the cities have neither seasonal patterns nor geographical variations in common. Naranjo, though it has very humid years during the cold phase, doesn't demonstrate a marked correspondence during years of the warm phase. Palmares shows more consistence with the warm phase, meanwhile San Ramón, has higher correspondence with both phases.
\end{abstract}

KEY WORDS: Annual precipitation, ENSO, Cold phase, warm phase, geographical variation.

RÉSUMÉ: L'étude analyse l'effet de l'événement ENOS (El Niño/La Niña-Oscillation du Sud) sur les précipitations annuelles des villes de Naranjo, Palmares et San Ramón, situées sur l'extrémité ouest de la Dépression Tectonique Centrale du Costa Rica. Nous disposons d'une période de 30 ans pour la ville de Naranjo, de 28 pour Palmares et de 42 pour San Ramón. Les résultats montrent des précipitations annuelles moyennes basses lors des événements de El Niño, et plus élevées pendant La Niña. Cependant, il n'existe ni un modèle temporal ni une similitude dans l'espace entre ces trois villes. Ainsi, Naranjo présente des années très humides pendant la phase froide, mais pas de correspondance marquée lors des années de la phase chaude. À Palmares, les précipitations ont tendance à diminuer pendant El Niño, ce qui équivaut à des années sèches, tandis que San Ramón semble avoir une forte correspondance avec les deux phases.

\footnotetext{
${ }^{1}$ Docente e Investigador Académico, Sede Occidente, Universidad de Costa Rica. mquesada@so.ucr.ac.cr
} 
MOTS-CLÉS: Précipitation annuelle, ENOS, Phase froide, Phase chaude, variation géographique.

RESUMO: Investiga-se o efeito do acontecimento ENOS (El Niño/La Niña- Oscilação Sul ) na precipitação anual das cidades de Naranjo, Palmares y San Ramón situados no setor extremo oeste da Depresión Tectónica Central de Costa Rica. A cidade de Naranjo conta com um total de 30 anos, Palmares 28 e San Ramón 42. A amostragem durante a permanência do acontecimento "El Niño" oferece um promedio baixo anual das precipitações atmosféricas, enquanto que os acontecimentos de "La Niña" são mais elevados. A pesar de tudo isso não existe um padrão temporal nem semelhança espacial entre as referidas cidades, como acontece em Naranjo, onde os anos são muito húmidos durante a estação fria, mas não registra uma correspondência marcada nos anos da estação cálida. Em Palmares as precipitações atmosféricas diminuem nos anos secos durante El Niño, enquanto que, em San Ramón, parecer ser que tem alta correspondência nas duas fases.

PALAVRAS CHAVE: Precipitação anual, ENOS, Fase Fria, Fase Cálida, variante geográfica.

\section{INTRODUCCIÓN.}

La distribución espacial de la precipitación pluvial depende de la orientación y altitud del relieve. En las montañas existen a veces depresiones a las que se les llama pasos de vientos, que permiten la incursión de masas de aire cargadas de humedad de un sector de la montaña hacia el otro. A esta condición se le ha llamado efecto del derrame de nubosidad, que se produce, principalmente, en las cumbres de los macizos montañosos, cambiando de esta forma las condiciones atmosféricas a sotavento (BARRY and CHORLEY, 1999). En otras ocasiones, algunas nubes son desplazadas por ráfagas de viento desde el sector de barlovento (sitio donde pegan las masas de aire húmedo) a sotavento (sitio protegido de las masas de aire húmedo), incrementando las precipitaciones en los lugares más elevados y en faldas o valles.

Además, existen condiciones oceánicas atmosféricas que no sólo determinan la cantidad de precipitación en un lugar, sino que también condicionan el período de ocurrencia; éstas normalmente tienen un comportamiento irregular de un año a otro. El territorio donde se ubica Costa Rica, por ser un istmo, tiene una alta influencia marítima.

Esta posición ístmica, así como su posición geográfica, hacen que Costa Rica esté influenciado durante todo el año por los llamados vientos Alisios que tienen una dirección noreste. El aumento de la velocidad del flujo Alisio propicia la ocurrencia de lluvias orográficas en la vertiente Caribe, dado que el ascenso de las masas de aire a través del sistema orográfico es determinante en la condensación y posterior precipitación. En tanto, en la vertiente Pacífica se dan condiciones totalmente diferentes, pues las masas de aire que logran pasar a sotavento pierden su humedad y se vuelven secas, ocasionando de esta manera 
condiciones estables. Este es el principio por el que se produce la época seca. No obstante, la época lluviosa no siempre presenta condiciones similares; durante unos años es más corta o viceversa, lo cual está directamente ligado a condiciones oceánico atmosféricas.

La localización de Costa Rica entre el mar Caribe y el Océano Pacífico, así como su complejidad topográfica, hace que se produzcan diversas variaciones climáticas, específicamente en los promedios de precipitación anual, los cuales también se encuentran relacionados con el evento ENOS (El Niño, La NiñaOscilación del Sur). A pesar de que existen algunos estudios sobre este evento en Costa Rica (FERNÁNDEZ y RAMÍREZ, 1991; WAYLEN ET AL., 1996; WAYLEN y QUESADA, 2002), aún no se ha realizado una investigación de carácter regional donde se mida su variabilidad.

\section{VARIABILIDAD CLIMÁTICA Y EL ENOS}

En los últimos veinte años han surgido varios estudios a escala mundial y regional que demuestran que existe una relación entre la ocurrencia y los aumentos o disminuciones en los promedios de la precipitación y el calentamiento de las aguas en el Océano Pacífico Ecuatorial (ROPELEWSKI AND HALPERT, 1987; ROGERS, 1988; WAYLEN ET AL, 1996; WAYLEN Y QUESADA, 2002).

Estas anomalías provocan que durante unos años se produzcan condiciones meteorológicas extremas, como sequías, inundaciones, nevadas, escarcha, etc., eventos que no son ocasionales, dado que provocan alteraciones drásticas en el clima (GLANTZ, 1996). Tales alteraciones han sido atribuidas a los cambios en las temperaturas superficiales del Océano Pacífico, a lo que se le llama El Niño(E.N) y La Niña (L.N) y a cambios en la presión atmosférica en el nivel del mar, también en el Océano Pacífico, a lo que se le ha dado el nombre de Oscilación del Sur (O.S). Estos cambios combinados han llegado a conocerse en el lenguaje popular como el evento El Niño y en el científico como el ENOS.

El evento El Niño puede describirse como la aparición no periódica de agua caliente en la superficie del mar en el Océano Pacífico Central y Oriental, cerca del Ecuador, pero que influye en otras regiones a nivel mundial, mostrando diversas circunstancias como son excesos o déficit de lluvia, nevadas, etc. La literatura popular y científica indican que la expresión El Niño (literalmente El Niño Jesús) fue usada por los pescadores peruanos, quienes observaban la aparición en ciertos años de agua caliente a lo largo de la costa de su país durante los periodos de navidad y que les afectaba la pesca (CAVIEDES, 1984). Actualmente, los científicos creen que el evento ENOS está relacionado con extremos anómalos en las condiciones meteorológicas en varias regiones del globo (HASTENRATH, 1967, 1978, 1984, 2002; ROPELEWSKI and HALPERT, 1987, 
1989, 1996; WAYLEN ET AL, 1996; ENFIELD, 1996; ENFIELD and MAYER, 1997; Chen ET AL, 1997; NARANJO, 1997; Alfaro ET AL, 1998).

La relación entre los eventos ENOS y los cambios inusuales en los patrones del clima mundial, ha sido denominada por científicos como teleconexiones. Al evento El Niño (E.N.) se le denomina la fase cálida al darse un calentamiento en las aguas oceánicas superficiales en las cercanías de las costas de Perú y Ecuador. En tanto, los eventos fríos a los cuales se les ha llamado La Niña (L.N.) completan el ciclo de ENOS. Durante esta fase disminuyen en unos cuantos grados centígrados las temperaturas superficiales del océano. Por lo general, los episodios fríos producen condiciones meteorológicas contrarias a las que produce la fase cálida. Los procesos que se desarrollan en el Océano Pacífico son también opuestos a los que se llevan a cabo durante la fase cálida del ENOS.

Por ejemplo, pareciera que existe una asociación entre los eventos ENOS y las sequías en la vertiente Pacífica de Costa Rica y con el aumento de las lluvias en la vertiente Caribe (WAYLEN y QUESADA, 2002), así como con la intensidad de los huracanes tropicales, dado que no en todos los años, tanto la intensidad como el número de huracanes se comportan igual (GRAY, 1993).

En Costa Rica la variabilidad topográfica es determinante en las diferencias climáticas y, sobre todo, en los promedios anuales de precipitación en el nivel regional. Unido a esto, existen patrones de circulación atmosféricos que influyen sobre el territorio nacional estacionalmente. Similarmente, la Zona de Convergencia Intertropical (ZCIT), que es una amplia faja de bajas presiones ubicada cerca del ecuador, la cual produce fuertes precipitaciones, especialmente sobre la vertiente Pacífica de Costa Rica durante una parte del año (BRENES y SABORIO, 1984). Por ejemplo, durante el verano boreal, la ZCIT supera la latitud a la que se encuentra ubicada Costa Rica, produciendo fuertes precipitaciones. Sin embargo, durante una parte del año ésta migra hacia el Sur y las precipitaciones disminuyen (HASTENRATH, 1967, 2002).

En síntesis, el régimen y la distribución de la precipitación están determinados por factores de índole topográfica, atmosférica y oceánica. En el caso de la región en estudio, interactúan todas estas condiciones y todo apunta a que también existen elementos de mesoescala (carácter regional) que hacen que sea uno de los sectores del país con presencia de un patrón de precipitación complejo.

\section{3. ÁREA DE ESTUDIO Y DATOS}

Las ciudades de Naranjo, Palmares y San Ramón se ubican en su extremo Oeste de la Depresión Tectónica Central de Costa Rica. Las tres ciudades están ubicadas muy cerca unas de otras (de 5 a $10 \mathrm{~km}$.). A pesar de su proximidad entre ellas, existen desigualdades en el relieve, dada la existencia de valles, coli- 
nas y serranías, lo que posiblemente marca diferencias en sus promedios de precipitación anual y estacional. Esta situación, la mayoría de las veces es muy marcada, aún a simple vista, con sólo movilizarse por la carretera que comunica las tres ciudades; al pasar de una serranía a otra, en un sector esta seco mientras que en el otro lado esta lloviendo.

La ciudad de Naranjo se encuentra a una altitud de 1042 m., y se ubica en las faldas de la sección volcánica central, mientras que Palmares con una altitud de $1017 \mathrm{~m}$., se ubica sobre una antigua caldera volcánica y está protegida en todos sus alrededores por colinas y serranías. La ciudad de San Ramón ubicada a una altitud de $1016 \mathrm{~m}$. pese a encontrarse en un valle, está expuesta a la penetración de masas de aire que normalmente incursionan por los pasos del Arenal y de Tapesco, quedando de esta forma no sólo expuesta a los vientos Oestes Ecuatoriales que provienen del Oeste, sino también de las masas provenientes del sector Caribe (FIGURA 1).

\section{MÉTODOS DE ANÁLISIS DE ENOS EN EL ÁREA EN ESTUDIO}

En esta investigación se han aplicado varias técnicas estadísticas para estimar la variación en la precipitación anual durante las fases del ENOS. Por el hecho de ser la lluvia una variable no constante se ha aplicado la siguiente prueba de distribución de probabilidad con la idea de tratar de medir su ocurrencia; WAYLEN and CAVIEDES (1990) en una investigación en la cuenca del río Aconcagua aplicaron la siguiente función:

$$
F(X \geq X)=\int_{-\infty}^{X} 1 / \sqrt{2 \pi \sigma} \cdot e^{-\left[(s-\mu)^{2} / 2 \sigma^{2}\right]} \cdot d s=N\left(\mu, \sigma^{2}\right)
$$

donde $\mu$ y $\sigma$ son el promedio y la variancia de la población respectivamente.

La distribución normal o Gaussian es adecuada para representar tanto períodos secos como lluviosos y se ajusta bien a períodos de precipitación anual. WAYLEN y CAVIEDES (1990) han ajustado esta distribución para el estudio de ENOS en su investigación, considerando las tres fases de la siguiente forma:

$$
F(X \geq X)=p_{1} \cdot\left[N\left(\mu_{1}, \sigma_{1}^{2}\right)\right]+p_{2} \cdot\left[N\left(\mu_{2}, \sigma_{2}^{2}\right)\right]+p_{3} \cdot\left[N\left(\mu_{3}, \sigma_{3}^{2}\right)\right]
$$

La precipitación anual de cada una de las fases se suma y de ahí se obtiene el promedio, la variancia y se analiza el comportamiento de la fase cálida (El Niño), la fase fría (La Niña) y la fase neutral (años normales). En esta oportunidad 
se utilizó la clasificación del ENOS del Center for Ocean-atmospheric Prediction Studies (http://www.coaps.fsu.edu/lib/elninolinks).

Se aplicó una prueba de distribución modificada Kolmogorov-Smirnov con un nivel de confianza del 0,05 . Tanto el promedio como la variancia se calculó para cada una de las tres condiciones oceánicas atmosféricas, de modo que cada registro se obtuviera para por medio de una prueba $t$ con dos colas con la idea de aprobar o rechazar una hipótesis nula. La prueba $t$ se usó para verificar las diferencias en los promedios y las variancias con la idea de visualizar si los registros de lluvias tienen una distribución normal.

(3)

(4)

$$
t_{\text {calc }}=\frac{\left(\overline{X_{1}}-\overline{X_{2}}\right)-D_{0}}{\sqrt{S_{p}^{2}\left(\frac{1}{n_{1}}+\frac{1}{n_{2}}\right)}}
$$

$$
S_{p}^{2}=\frac{\left(n_{1}-1\right) S_{1}^{2}+\left(n_{2}-1\right) S_{2}^{2}}{\left(n_{1}-1\right)+\left(n_{2}-1\right)}
$$

(5)

$$
=\frac{\left(n_{1}-1\right) S_{1}^{2}+\left(n_{2}-1\right) S_{2}^{2}}{n_{1}+n_{2}-2}
$$

(6)

$$
t_{c r i t}=\left(W_{1} t_{1}+W_{2} t_{2}\right) /\left(W_{1}+W_{2}\right)
$$

donde

$$
\begin{gathered}
W_{i}=S_{i}^{2} / n_{i} \\
t_{i}=t_{1-\alpha / 2, n_{i}-1}
\end{gathered}
$$

La prueba estadística de combinación de promedios y variancias se aplicó con el fin de buscar asociaciones extremas entre las fases y se obtuvieron porcentajes que indican si el registro de precipitación tiene correspondencia con años $\mathrm{La}$ Niña o con años El Niño. La significancia estadística de la relación en L.N y E.N event ${ }^{\circ}$ os y la precipitación anual se examina utilizando una distribución hipergeométrica, donde se pueden obtener episodios secos o húmedos. 


\section{IDENTIFICACIÓN DE LOS AÑOS DE OCURRENCIA DE LOS EVENTOS EL NIÑO Y LA NIÑA}

En el CUADRO 1 se muestra la subdivisión en años La Niña, años normales y años El Niño, la cual ha sido aplicada a cada una de las estaciones climatológicas ubicadas en las ciudades en estudio y de acuerdo con el registro de precipitación existente en cada una de ellas. Esto con la idea de visualizar la aparición de cada una de las fases, encontrándose que en los tres lugares se han manifestado las distintas fases o episodios del ENOS.

Cada una de las fases comprende años distintos, los cuales durante ciertos períodos se extienden por varios años, existiendo un predominio de años normales, en tanto que los años de El Niño son los menos frecuentes. Además, del CUADRO 1 se puede extraer que no existe una periodicidad marcada en cuanto a la ocurrencia de las fases.

Cuadro 1. Clasificación anual de las fases del evento ENOS

\begin{tabular}{ccc}
\hline fase fría & fase neutral & fase caliente \\
\hline 1942 & 1941 & 1940 \\
1944 & 1943 & 1951 \\
1945 & 1950 & 1957 \\
1946 & 1952 & 1963 \\
1947 & 1953 & 1965 \\
1948 & 1958 & 1969 \\
1949 & 1959 & 1972 \\
1954 & 1960 & 1976 \\
1955 & 1961 & 1982 \\
1956 & 1962 & 1986 \\
1964 & 1966 & 1987 \\
1967 & 1968 & 1991 \\
1970 & 1974 & 1997 \\
1971 & 1977 & \\
1973 & 1978 & \\
1975 & 1980 & \\
1979 & 1981 & \\
1998 & 1983 & \\
1999 & 1984 & \\
& 1985 & \\
& 1989 & \\
& 1990 & \\
& 1992 & \\
& 1993 & \\
\hline
\end{tabular}

Fuente: http://www.coaps.fsu.edu/lib/elninolinks 
En el CUADRO 2 se observa que la precipitación disminuye durante la fase cálida, en tanto que durante la fase fría se incrementa. Por ejemplo, para la ciudad de Naranjo se dan aumentos en la precipitación de hasta un 18\% durante los años La Niña, mientras que en la ciudad de Palmares más bien la precipitación decrece en un 12\% durante los años El Niño. Similarmente, en San Ramón se dan aumentos de la precipitación en un 29\% durante años El Niño. Mientras tanto, durante los años normales se mantiene la precipitación con valores intermedios.

Cuadro 2. Promedios y desviaciones estándars de la precipitación anual para las ciudades en estudio en las diferentes fases $(\mathrm{mm})$

\begin{tabular}{lrrrrrrrrr}
\hline Estación & \multicolumn{3}{c}{ Normal } & \multicolumn{4}{c}{ La Niña } & \multicolumn{3}{c}{ El Niño } \\
\cline { 2 - 10 } & $A$ & $B$ & $C$ & $A$ & $B$ & $C$ & $A$ & $B$ & $C$ \\
\hline Naranjo & 14 & 2184,3 & 396,9 & 9 & 2548,1 & 316,1 & 7 & 2082,5 & 268,4 \\
Palmares & 14 & 1865,3 & 351,7 & 6 & 1987,8 & 301,4 & 8 & 1774,0 & 385,4 \\
San Ramón & 23 & 1841,0 & 357,3 & 10 & 2255,6 & 366,1 & 9 & 1596,6 & 305,1 \\
\hline
\end{tabular}

A, años; B, Promedio; C, Desviación Standard

FUENTE: Elaboración propia

Cuadro 3. Niveles anuales de precipitación y promedios durante las fases del ENOS para cada ciudad en estudio

\begin{tabular}{lrrrr}
\hline & Nivel $(\mathrm{mm})$ & Fase Fría & Fase Normal & Fase Caliente \\
\hline NARANJO & & & & \\
Húmeda & 2567,1 & 67,9 & 24,5 & 13,5 \\
Normal & & 24,1 & 37,2 & 34,4 \\
Seca & 2229,5 & 8,0 & 38,3 & 52,1 \\
\hline PALMARES & & & & \\
Húmeda & 2057,1 & 67,3 & 32,1 & 9,0 \\
Normal & & 26,5 & 35,2 & 31,2 \\
Seca & 1729,8 & 6,2 & 32,7 & 59,8 \\
\hline SAN RAMON & & & & \\
Húmeda & 2073,3 & 62,1 & 30,4 & 11,6 \\
Normal & & 28,0 & 38,6 & 28,8 \\
Seca & 1719,1 & 9,9 & 31,0 & 59,6 \\
\hline
\end{tabular}

FUENTE: Elaboración propia

En el CUADRO 3 se muestra de la variabilidad en las fases del ENOS, sobre todo durante su fase fría, donde existe una probabilidad mayor al 60 por ciento de que las tres estaciones obtengan un período lluvioso y menos de un 10 por ciento de obtener períodos secos. Por ejemplo, durante la fase fría la proporción de los promedios anuales húmedos fue de un 67,9 por ciento para Naranjo, un 67,3 por ciento para Palmares y un 62,1 por ciento para San Ramón, lo cual demuestra que los mayores promedios de precipitación se producen durante la fase fría, siendo la localidad de Naranjo la que muestra un porcentaje más alto. En tanto, 
durante la fase cálida existe una alta probabilidad de obtener más de un 50 por ciento de que se presente un año seco y menos de un 14 por ciento de probabilidad de que sea un año lluvioso. Un ejemplo, lo constituye el 52,1 por ciento para Naranjo, un 59,8 por ciento, para Palmares y un 59,6 por ciento para San Ramón de que se registren años secos.

Una forma de tratar de visualizar posibles diferencias regionales en la precipitación anual entre las tres ciudades en estudio durante las fases del ENOS, consiste en comparar las poblaciones de las variancias entre las tres estaciones. Ésta se hace por medio del método Prueba F Fisher (LEYTHAM, 1984), que es usado para probar una hipótesis alternativa de que la población de las variancias difieren entre sí, o para comprobar una hipótesis nula de que ambas poblaciones de variancias son iguales. Mientras que cuando las poblaciones de variancias no son iguales los resultados muestran una $\mathrm{F}$ grande o pequeña. En el caso de las ciudades en estudio se lograron los siguientes resultados.

Cuadro 4. Tasas de la prueba F para las tres condiciones oceánico atmosféricas

\begin{tabular}{|c|c|c|c|c|c|c|}
\hline & \multicolumn{2}{|c|}{ Normal / La Niña } & \multicolumn{2}{|c|}{ Normal / El Niño } & \multicolumn{2}{|c|}{ La Niña / El Niño } \\
\hline & $A$ & $B$ & $A$ & $B$ & $A$ & $B$ \\
\hline Naranjo & 1,55 & 2,31 & 1,69 & 2,31 & 1,09 & 2,60 \\
\hline Palmares & 2,15 & 2,46 & 6,12 & 2,77 & 2,86 & 2,91 \\
\hline San Ramón & 1,18 & 2,65 & 1,49 & 2,38 & 1,77 & 2,85 \\
\hline
\end{tabular}

A, F tabulado; $b, F$ crítico

FUENTE: Elaboración propia

En el CUADRO 4 a pesar de la exactitud de la prueba, sólo se visualiza un ligero cambio durante la relación años normales con años El Niño en la estación de Palmares, lo que pareciera indicar que es en este lugar donde El Niño es más intenso, el resto de las combinaciones no muestran mayores diferencias. Por ello es necesario aplicar otra prueba que considere no sólo las variancias sino también los promedios de precipitación anual juntos.

\subsection{Diferenciación de años extremos de precipitación}

CAVIEDES y WAYLEN (1991) crearon un proceso para combinar variancias y promedios con la idea de identificar diferencias regionales bajo las tres fases del ENOS. Estos investigadores asumieron que el total de precipitación anual para cada una de las fases puede ser modelado bajo una distribución normal, combinando promedios y variancias; de esta forma obtuvieron nueve posibilidades, las cuales se detallan a continuación:

I. Los tres promedios y variancias son diferentes. Lo que significa que la cantidad de precipitación en las tres fases es diferente. 
II. Dos promedios son iguales y las tres variancias son diferentes. En este caso El Niño o La Niña podrían tener diferentes promedios de precipitación.

III. Los tres promedios son similares pero las tres variancias son diferentes. Esto implica que las variaciones en la precipitación en cada subpoblación pueden variar.

IV. Todos los promedios son diferentes y dos variancias son iguales. Lo que demuestra que llueve muy parecido entre las fases pero en dos de ellas la cantidad de precipitación ocurre en forma irregular.

V. Un promedio es diferente (ya sea El Niño, La Niña o los años normales) y dos de las variancias son similares. En una de las fases llueve más o menos que en las otras.

VI. Todos los promedios son iguales y una de las variancias es diferente. Esa variancia puede ser un año normal, El Niño o La Niña.

VII. Todos los promedios son diferentes y las variancias son iguales. Lo que demuestra que no hay diferencias regionales en cuanto a la cantidad de precipitación.

VIII. Un promedio es diferente pero todas las variancias son similares. En alguna de las fases existe algún elemento que hace que la precipitación varíe.

IX. Todos los promedios y las variancias son similares. Esto demuestra que no hay ninguna diferencia entre las fases.

Aunque se espera que en los años de La Niña o El Niño se muestren valores altos o bajos en los promedios de precipitación anual, también los años normales a veces muestran valores altos o bajos, por el hecho de que aún en estos años suceden disturbios atmosféricos, como es el caso de ondas tropicales, cambios en el patrón de circulación de los vientos, entre otros, que provocan valores altos o bajos en el promedio de precipitación.

Cuadro 5. Promedios y variancias altas durante las tres fases oceánico-atmosféricas para las tres ciudades en estudio

\begin{tabular}{|c|c|c|c|c|c|c|}
\hline \multirow[b]{2}{*}{ Ciudad } & \multicolumn{3}{|c|}{ Promedio alto } & \multicolumn{3}{|c|}{ Variancia alta } \\
\hline & Normal & La Niña & El Niño & Normal & La Niña & El Niño \\
\hline Naranjo & & घ & & - & & \\
\hline Palmares & & - & & & & - \\
\hline San Ramón & & [ & & & - & \\
\hline
\end{tabular}

FUENTE: Elaboración propia

Del anterior análisis de las estaciones en estudio se encontraron los siguientes resultados: 
1. Palmares: Se ubica en el grupo IV, Esto demuestra que los años más lluviosos son durante los años de La Niña, mientras que los años que muestran mayores variaciones son los años de El Niño.

2. Naranjo: Se ubica en el grupo $\mathrm{V}$, indicando que los promedios más elevados se dan durante los años La Niña y la mayor variabilidad durante los años normales. De esta forma, la ciudad de Naranjo es por excelencia un lugar lluvioso y es durante los años normales que se produce una mayor variabilidad de la precipitación.

3. San Ramón: Se ubica también en el grupo V, tanto los mayores promedios de precipitación como sus variancias se producen durante los años de La Niña. La posición geográfica de esta ciudad provoca que sea muy vulnerable a la penetración y a los cambios en la velocidad del viento proveniente tanto del sector Pacífico como del Caribe, lo que justifica que esta región sea muy inestable climáticamente, repercutiendo en la cantidad de lluvia que cae sobre dicha ciudad.

Estas tres ciudades, a pesar de su cercanía, muestran elementos geográficos y climatológicos propios que propician una gran variabilidad en la precipitación anual como se ha demostrado en el anterior análisis, lo que también se puede reafirmar al comparar los porcentajes de cambio en los promedios anuales de precipitación.

\subsection{Porcentaje de cambio en los promedios anuales de precipitación}

El CUADRO 6 detalla los cambios en los promedios anuales de precipitación entre los años El Niño y La Niña para las tres ciudades en estudio.

\begin{tabular}{|c|c|}
\hline & \\
\hline Palmares & \\
\hline Palmares & 10,7 \\
\hline Naranjo & 18,3 \\
\hline San Ramón & 29,2 \\
\hline
\end{tabular}

FUENTE: Elaboración propia

Los valores más elevados indican que existe un mayor porcentaje de disminución de la precipitación durante los años de El Niño con respecto a los años de La Niña y cuando los valores son cercanos a cero demuestran que existe una mayor similitud en los porcentajes de lluvia durante los años El Niño y La Niña. El mayor porcentaje de cambio sucede en las ciudades de San Ramón y Na- 
ranjo, mientras que en Palmares se registra un porcentaje de disminución inferior. Esto es una muestra de que tanto en San Ramón como en Naranjo los años más lluviosos se dan durante La Niña, mientras que en Palmares, los años de La Niña no parecieran ser tan lluviosos. Más bien, existe una mayor tendencia a que se registren años secos durante El Niño. En la ciudad de San Ramón pareciera que existe una influencia más marcada a que se registren unos años lluviosos y otros secos, aspecto que no se visualiza en las dos restantes ciudades en una forma tan marcada.

\section{CONCLUSIÓN}

Como reflexión final, se obtiene que las pruebas estadísticas aplicadas proveen un buen análisis de los registros de precipitación anual existentes en las tres ciudades que se han estudiado, que a pesar de su proximidad, se aprecian diferencias regionales marcadas de un año a otro e incluso en cuanto a la cantidad de lluvia que cae sobre cada una de ellas. Se demuestra así que las diferencias en la precipitación regional en el sector oeste de la Depresión Tectónica Central no sólo son temporales sino que también regionales.

De acuerdo con los resultados de la investigación, existe una mayor probabilidad de que ocurran promedios bajos de precipitación anual durante los años El Niño y promedios altos durante los años La Niña. Pareciera que la fase cálida es más marcada en aquellos lugares de topografía plana, en valles y que tienen una mayor influencia marítima y de corrientes de viento, en los casos de Palmares y San Ramón.

En aquellos lugares con suelos irregulares y con rasgos de un clima tipo continental, la fase cálida del ENOS es menos consistente; un ejemplo típico lo constituye la ciudad de Naranjo, dada su ubicación en un área muy irregular topográficamente y aunado a que es una de las pocas ciudades de Costa Rica con un clima continental. Estos factores facilitan que la disminución de los promedios de precipitación anual sea menos pronunciada durante los años de El Niño. En tanto que San Ramón, por su ubicación expuesta a pasos de vientos, se encuentra bajo condiciones muy inestables climáticamente, como se señaló anteriormente, teniendo una respuesta positiva, tanto ante La Niña como ante El Niño.

En síntesis, este estudio provee suficientes evidencias cuantitativas para probar la hipótesis que el patrón de precipitación interanual es muy variable en Costa Rica debido a la influencia del evento ENOS. Aunado a esto, se ha demostrado que aún en pequeños sectores como el que se estudió se pueden encontrar diferencias marcadas. 


\section{REFERENCIAS}

ALFARO ET AL (1998). «Relaciones entre la precipitación en Centroamérica y los Océanos Pacífico y Atlántico Tropical». Investigaciones marinas, 26, 59-69.

BARRY, R. and CHORLEY, R. (1999). Atmosphere, Weather and Climate. Sixth Ed. Chapman and Hall Inc. Routledge, NY.

BRENES, A. y SABORÍO, V. (1984). «Changes in the general circulation and influence on precipitation trends in Central America: Costa Rica». En Ambio. Vol. 23. 23(1): 87-90.

CAVIEDES, C. (1984). «El niño 1982-1983». American Geographical Society. New York. Vol 74. pp 288-289.

CAVIEDES, C., and WAYLEN, P. (1991) «Chapters for a climatic history of South America», W. 1 texto, Endlicher and H. Gossmann, editors, Beitrage Zur Regionalen and Angewand ten Rlimatologie, Freiburger Geogrphische Hefte, vol. 32. Pp.149-180.

CHEN, A. ET AL.(1997). «Using SST anomalies to predict flood and drought conditions for the Caribbean». Center for Ocean-Land-Atmosphere Studies Report, 49.

ENFIELD, D. (1996). «Relationship of inter-American rainfall to tropical Atlantic and Pacific SST variability». Geophys. Res. Letters. 23, 3305-3308.

ENFIELD, D., and MAYER, D. (1997). «Tropical Atlantic sea surface temperature variability and its relation to El Niño-Southern Oscillation». J. Geophys. Res., 102. 929-945.

FERNÁNDEZ, W.y RAMíREZ, P. (1991). «El Niño, La Oscilación del Sur sus efectos sobre Costa Rica: Una revisión». Tecnología en Marcha 11:3-11

GLANTZ, M. (1996). Corrents of change El Nino's impacts on climate and society. $1^{a}$ edición. Cambriddge. Cambriddge University Press.

GRANDOSO, H. y OTROS. (1982). «Características de la atmósfera libre sobre Costa Rica y su relación con la precipitación». Informe semestral, I.G.N, Costa Rica. Enero-Junio, pp. 11-52. San José, Costa Rica.

GRAY, C. (1993). «Regional Meteorology and Hurricans». Climate Change in the Intra- American Sea . G.Maul, Ed, Eduard Arnold, 112. 87-99.

HASTENRATH, S. (1967). «Rainfall distribution and regime in Central America». Archiv fur Meteorologie, Geophysik und Bioklimatologie, Serie B Band 15, Heft 3: 201-241.

HASTENRATH, S. (1978). «On the modes of tropical circulation and climate anomalies». J. Atmosphere Science. 35. 2222-2231.

HASTENRATH, S. (1984). «Interannual variability and annual cycle: mechanisms of circulation and climate in the tropical Atlantic sector». Monthy Weather Review. 112. 1097-1107. 
HASTENRATH, S. (2002). «The Inertropical Convergence Zona of the Eestern Pacific Reviseted», International Journal of Climatology. 22: 347-356. http://www.coaps.fsu.edu/lib/elninolinks

LEYTHAM, K.(1984). «Maximum likelihood estimates for the parameters of mixture distributions». Water Resources research. Vol. 20. pp. 896-1588.

NARANJO, L., (1997). Impacts of ENSO on Cuba. Contribution to "A systems approach to ENSO", A colloquium on El Niño-Southern Oscillation: Atmosphere, Oceanic, Societal Environmental and Policy Perspectives. Colorado, United States of America.

NichOLLS, J. (1987). «The El Niño/ Southern Oscillation phenomenon». In: Climate crisis. Ed. M.H. UN Publications. New York: 2-10.

ROGERS, J. (1988). «Precipitation Variability over the Caribbean and Tropical Americas Associated with the Southern Oscillation», En: Journal of Climate, 1, , 172-182.

ROPElEWSKI, C., HALPERT, M. (1987). «Global and Regional Precipitation Patterns Associated with the El Niño|Southern Oscillation». En: Monthly Weather Review, 115, 606-1626.

WAYLEN, P., CAVIEDES, C. (1990). «Annual and seasonal fluctuations of precipitation and streamflow in the Aconcagua river basin, Chile». Journal of Hydrology. 120. 79-193.

WAYLEN, P., CAVIEDES, C y QuESADA, M. (1996). «Interannual Variability of Monthly Precipitation in Costa Rica». En: Journal of Climate, 9, 2606-2613.

WAYLEN, P y QUESADA, M. (2002). «The effect of Atlantic and Pacific sea surface temperature on the Mid-Summer drought of Costa Rica». Environmental change and weather sustainability. Instituto Pirenaico de Ecología, Zaragoza. España. 197-209. 
Figura 1. Ubicación ciudades Naranjo, Palmares y San Ramón, Costa Rica

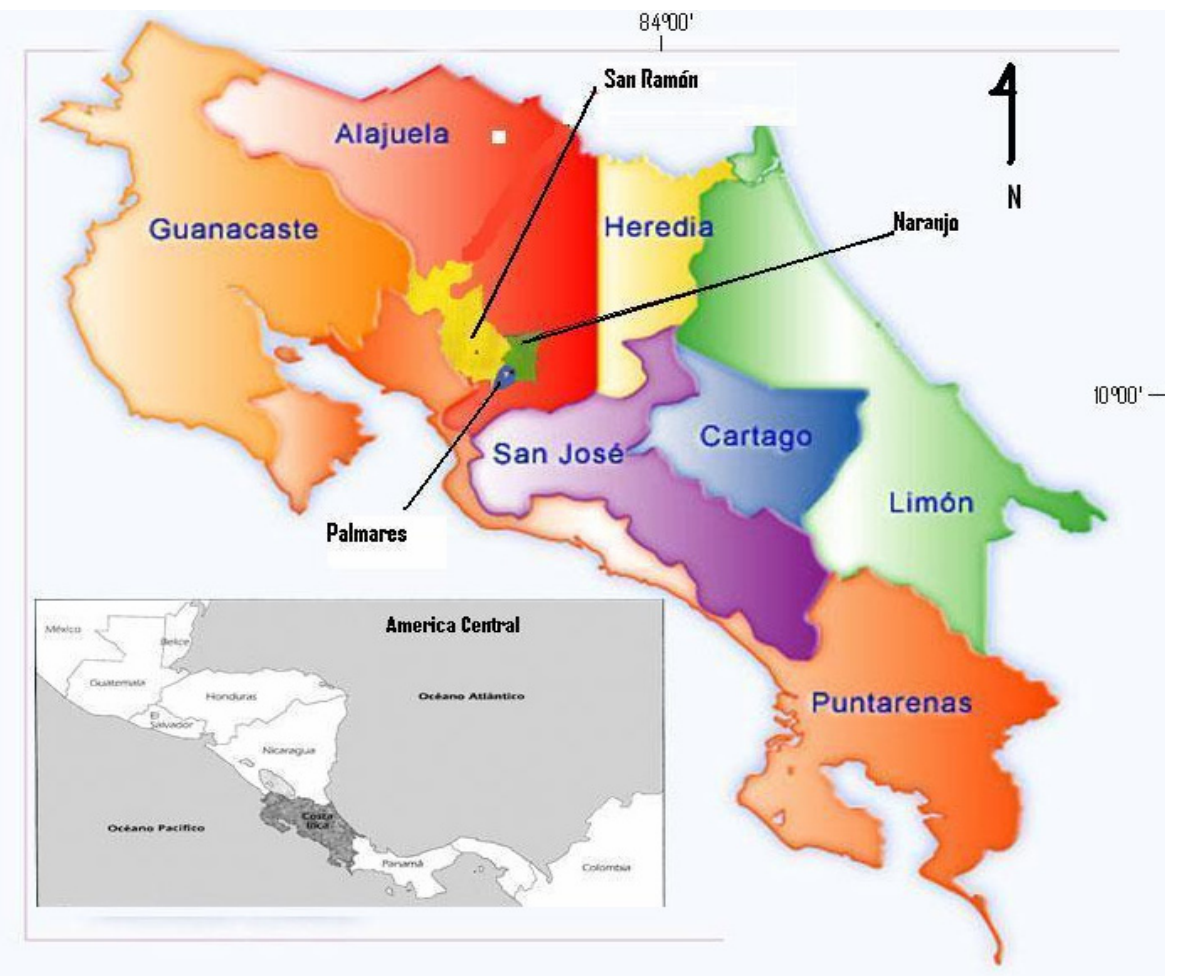

ELABORÓ: Oscar Salazar Sancho, 2006. 